Beata Śmigielska

Université de Silésie, Katowice

Pologne

(D) https://orcid.org/0000-0002-3383-0030

\section{Cadres, scripts, registres - complexité de traduction des mots polysémiques}

Frames, scripts, registers -

complexity of translation of polysemic words

\begin{abstract}
The article deals with issues related to the description of polysemic units for supervised automatic translation of texts from French to Polish based on the object-oriented method by W. Banyś (AOO). Applying to the description additional tools allowing for the disambiguation - frames, scripts and register analysis - the author focuses on presenting the complexity of the description of lexical units. It should be stressed that the decision to assign meanings of such words one or another equivalents in the target language is determined by the criterion of preference. Some of the meanings of the word are easy to describe in such a way that the program can make a correct translation into the target language with a high degree of probability. However, there are often such meanings whose description is much more complex. The closer the meanings of the disambiguated words in the original and target language and the more similar the communication situation in which they are used, the more difficult it is to clearly indicate the boundaries between the meanings. In such cases, there is a much greater range of preferential character of the translation, which we have to adopt The French noun conjunction ( $f$ ), chosen for analysis, reflects this phenomenon very well.
\end{abstract}

\title{
Keywords
}

Object oriented approach, supervised automatic translation, operators, attributes, frames, scripts, registers

Nous allons discuter brièvement dans ce texte le problème de la complexité de description sémantique des mots polysémiques dans le cadre du traitement automatique assisté des textes en basant sur l'Approche Orientée Objets de W. B a n y ś 
(2002a, 2002b, 2005, 2018). Les outils qui nous permettront d'atteindre le but visé sont les cadres et les scripts grâce auxquels il est possible de délimiter des frontières nécessaires entre des significations particulières des mots en attribuant en même temps à chacune d'elles un équivalent adéquat dans la langue cible. Il faut rappeler ici que les cadres et les scripts dans l'AOO sont compris comme des situations de communication où un mot ou une expression donnés apparaît. Ce sont tous les éléments nécessaires, statiques - cadres et dynamiques - scripts, qui créent telle ou autre situation activant l'un des sens d'une expression polysémique. C'est grâce à ces éléments-là, nous, les humains, décodons les messages (cf. p. ex. A. Czekaj, 2011, 2018 ; M. Hrabia, 2016 ; B. Śmigielska, 2013, 2016, 2017 ; à propos des frames et des scripts, voir aussi p. ex. Ch. Fi $11 \mathrm{~m}$ or e, 1982 ; E. G offma n, 2010 ; M. Minsky, 1985 ; Z. Kövecses, 2011 ; R. S c hank, R. Abels on, 1977).

Dans ce qui suit nous voudrions soumettre à l'analyse le substantif français conjonction $(f)$ qui nous servira d'exemple pour illustrer le processus de désambiguïsation sémantique des mots mettant en relief la complexité des relations entre ses sens. L'exemple choisi permettra de rendre compte, d'un côté, du fait que certains sens des mots polysémiques ne sont pas difficiles à décrire à l'aide des outils mentionnés, et de l'autre côté, ce qui est encore plus important, du fait que les descriptions ainsi faites pourraient servir de base pour leur traduction automatique dans un contexte donné. Mais il existe aussi des sens dont les frontières précises ne sont ni évidentes ni faciles à tracer. Cependant, il faut tenir compte que la langue naturelle en tant que système vivant se développe sans cesse et ses locuteurs l'emploient de manière tellement créative qu'il est même parfois impossible de prévoir tous les emplois éventuels des mots (cf. p. ex. A. Kilgariff, 1997). Ainsi, la description du niveau sémantique des unités linguistiques, surtout polysémiques, exige souvent l'application de l'approche du type préférentiel (c'est-à-dire qu'on propose plus ou moins telle ou telle traduction du mot ou de l'expression dans un contexte donné basant davantage sur sa probabilité que sur une certitude absolue).

L'exemple que nous avons choisi nous paraît intéressant de ce point de vue, même si au premier coup d'œil il pourrait paraître ne pas l'être, ce qui montre une fois de plus qu'il ne faut pas se fier aux apparences. Commençons donc par la définition raccourcie du substantif conjonction $(f)$ dans le Trésor de la Langue Française informatisé TLFi (http://atilf.atilf.fr/, consulté le 25 avril 2020) :

CONJONCTION, subst. fém.

I. - Action de se conjuguer, de se joindre.

A. - Action de s'unir en vue de produire un effet précis.

1. [À propos de pers.] Union : 
- En partic. Union charnelle. Le but essentiel de ceux qui s'aiment est de créer et de connaître ensemble, par la conjonction physique et charnelle, l'élan vers la mort, vers la dépersonnalisation intense (THIBAUDET, Réflexions sur la litt., 1936, p. 67) $[\ldots]$

2. [À propos de choses concr.] Spéc., ASTRON. et ASTROL. Rencontre de deux ou plusieurs planètes en une ligne droite par rapport à un point de la terre. Les Chaldéens auraient pensé que le monde finissait tantôt par l'eau, tantôt par le feu suivant deux " grandes années » ou conjonctions diverses des planètes (LEROUX, De l'Humanité, t. 2, 1840, p. 700) [...]

3. [À propos de choses abstr.] Conjonction des efforts. Le monde et moi. (...) une conjonction qui se faisait mal, un accord qu'ils suaient sang et eau pour établir! (GUÉHENNO, Journal d'une « Révolution », 1938, p. 167). Le style d'un peintre est dans cette conjonction de la nature et de l'histoire (CAMUS, L'Homme révolté, 1951, p. 318) [...]

\section{B. - [À propos d'événements] Rencontre fortuite, coïncidence.}

Conjonction de fatalité (HUGO, Correspondance, 1855, p. 212). On voyagerait ainsi (...) pendant des centaines de siècles, que jamais on ne ferait les mêmes rencontres ni ne retrouverait les mêmes conjonctions de choses (GOBINEAU, Nouvelles asiatiques, La Vie de voyage, 1876, p. 333). Sentir dans les propos tout le poids de la coïncidence, de la conjonction d'événements (VALÉRY, Variété IV, 1938, p. 120) [...]

\section{II. - Ce qui sert à joindre.}

A. - GRAMM. Mot invariable qui a pour fonction de joindre deux mots, des groupes de mots.

Sa phrase pleine, claire, longue pourtant et perpétuellement enchaînée de l'une à l'autre par des conjonctions, n'avait pas encore tout à fait secoué le joug du latinisme (SAINTE-BEUVE, Port-Royal, t. 2, 1842, p. 547) [...]

\section{B. - RHÉT. Répétition du même mot invariable reliant les différentes parties d'une période pour produire un effet d'insistance [...].}

Après avoir analysé la définition ci-dessus, nous pouvons immédiatement distinguer trois équivalents polonais : połaczenie, koniunkcja et spójnik. Il semble, au premier abord, que deux derniers appartiennent aux domaines spécialisés (astronomie, astrologie, rhétorique, logique, grammaire), tandis que le premier est réservé plutôt à la langue générale.

Il est à remarquer que dans le cas du mot conjonction (f) traduit comme koniunkcja ou spójnik, il existe des mots dans leurs contextes immédiats, leurs attributs et opérateurs, qui activeront, à eux seuls, cette traduction-là (cf. p. ex. B. Ś m i g i el s k a, 2015, 2017). Dans un premier temps, il suffit d'observer leurs emplois et de lister tous ceux qui indiqueront de façon directe et en principe non ambiguë leurs équivalents polonais correspondants. 
Attributs :

conjonction $(f)$ - spójnik

\begin{tabular}{|l|l|}
\hline conjonction argumentative & spójnik argumentacyjny \\
\hline conjonction causale & spójnik przyczyny \\
\hline conjonction complexe & spójnik złożony \\
\hline conjonction composée & spójnik złożony \\
\hline conjonction copulative & spójnik łączny (kopulatywny) \\
\hline conjonction d'opposition & spójnik przeciwstawny \\
\hline conjonction de but & spójnik celu \\
\hline conjonction de cause & spójnik przyczyny \\
\hline conjonction de comparaison & spójnik porównania \\
\hline conjonction de condition & spójnik warunku \\
\hline conjonction de conséquence & spójnik konsekwencji \\
\hline conjonction de coordination & spójnik współrzędności \\
\hline conjonction de négation & spójnik negacji \\
\hline conjonction de subordination & spójnik podrzędności \\
\hline conjonction synonyme & spójnik synonim \\
\hline conjonction de temps & spójnik czasu \\
\hline conjonction disjonctive & spójnik rozłączny \\
\hline conjonction simple & spójnik prosty \\
\hline etc. & etc. \\
\hline
\end{tabular}

Opérateurs :

conjonction $(f)$ - spójnik

\begin{tabular}{|l|l|}
\hline ajouter la conjonction & dorzucić spójnik \\
\hline apprendre par cœur la conjonction & nauczyć się na pamięć spójnika \\
\hline effacer la conjontion & wymazać spójnik \\
\hline mettre la conjonction & wstawić spójnik \\
\hline placer la conjonction & umieścić spójnik \\
\hline etc. & etc. \\
\hline & \\
\hline conjonction associe qqch. à qqch. & spójnik łączy coś z czymś \\
\hline conjonction interconnecte qqch. & spójnik łączy coś \\
\hline conjonction conjoint qqch. & spójnik spaja coś \\
\hline conjonction disjoint qqch. & spójnik rozdziela coś \\
\hline conjonction joint qqch. à qqch. & spójnik łączy coś z czymśs \\
\hline conjonction lie qqch. à qqch. & spójnik łączy coś z czymś \\
\hline conjonction relie qqch. à qqch. & spójnik łączy coś z czymś \\
\hline conjonction se combine avec qqch. & spójnik łączy się z czymś \\
\hline conjonction sépare qqch. de qqch. & spójnik oddziela coś od czegoś \\
\hline conjonction unit qqch. à qqch. & spójnik łączy coś z czymś \\
\hline etc. & etc. \\
\hline
\end{tabular}


Attributs :

conjonction $(f)$ - koniunkcja (astrologia, astronomia, retoryka, logika)

\begin{tabular}{|c|c|}
\hline conjonction écliptique & koniunkcja ekliptyczna \\
\hline conjonction apparente & pozorna koniunkcja \\
\hline conjonction astrale & koniunkcja astralna \\
\hline conjonction astronomique & koniunkcja astronomiczna \\
\hline conjonction binaire & podwójna koniunkcja \\
\hline conjonction contradictionnelle & sprzeczna koniunkcja \\
\hline conjonction d'axiomes & koniunkcja aksjomatów \\
\hline conjonction des astres & koniunkcja gwiazd \\
\hline conjoinction de deux objets célestes & koniunkcja między dwoma ciałami niebieskimi \\
\hline conjonction de deux propositions & koniunkcja dwóch zdań \\
\hline conjonction des ensembles & koniunkcja zbiorów \\
\hline conjonction des étoiles & koniunkcja gwiazd \\
\hline conjonction des négations & koniunkcja zaprzeczeń \\
\hline conjonction des notions & koniunkcja pojęć \\
\hline conjonction des objets célestes & koniunkcja ciał niebieskich \\
\hline conjonction des phrases & koniunkcja zdań \\
\hline conjonction des planètes & koniunkcja planet \\
\hline conjonction des propositions & koniunkcja zdań \\
\hline conjonction des thèses & koniunkcja tez \\
\hline conjonction entre deux planètes & koniunkcja między dwoma planetami \\
\hline conjonction géocentrique & koniunkcja geocentryczna \\
\hline conjonction inférieure & koniunkcja dolna \\
\hline conjonction lente & powolna koniunkcja \\
\hline conjonction logique & koniunkcja logiczna \\
\hline conjonction lunaire & koniunkcja księżyca \\
\hline conjonction moyenne & koniunkcja środkowa \\
\hline conjonction Mars Neptune & koniunkcja Marsa i Neptuna \\
\hline conjonction périodique & koniunkcja okresowa \\
\hline conjonction planétaire & koniunkcja planet \\
\hline conjonction propositionnelle & koniunkcja zdań \\
\hline conjonction rhétorique & koniunkcja w retoryce \\
\hline conjonction solaire & koniunkcja słońca \\
\hline conjonction supérieure & koniunkcja górna \\
\hline double conjonction & podwójna koniunkcja \\
\hline Grande conjonction & Wielka koniunkcja \\
\hline fausse conjonction & fałszywa koniunkcja \\
\hline prochaine conjonction & najbliższa koniunkcja \\
\hline triple conjonction & potrójna koniunkcja \\
\hline vraie conjonction & prawdziwa koniunkcja \\
\hline etc. & etc. \\
\hline
\end{tabular}


Cf. aussi la concordance générale, sans délimiter différents sens du mot, de conjonction $(f)$ dans Sketch Engine https://app.sketchengine.eu/\#wordsketch?cor pname $=$ preloaded $\% 2$ Ffrtenten $17 \_$fl2\&tab $=$basic\&lemma $=$conjonction\&showresul ts $=1$ (consulté le 25 avril 2020) :

\section{WORD SKETCH}

French Web 2017 (frTenTen17)

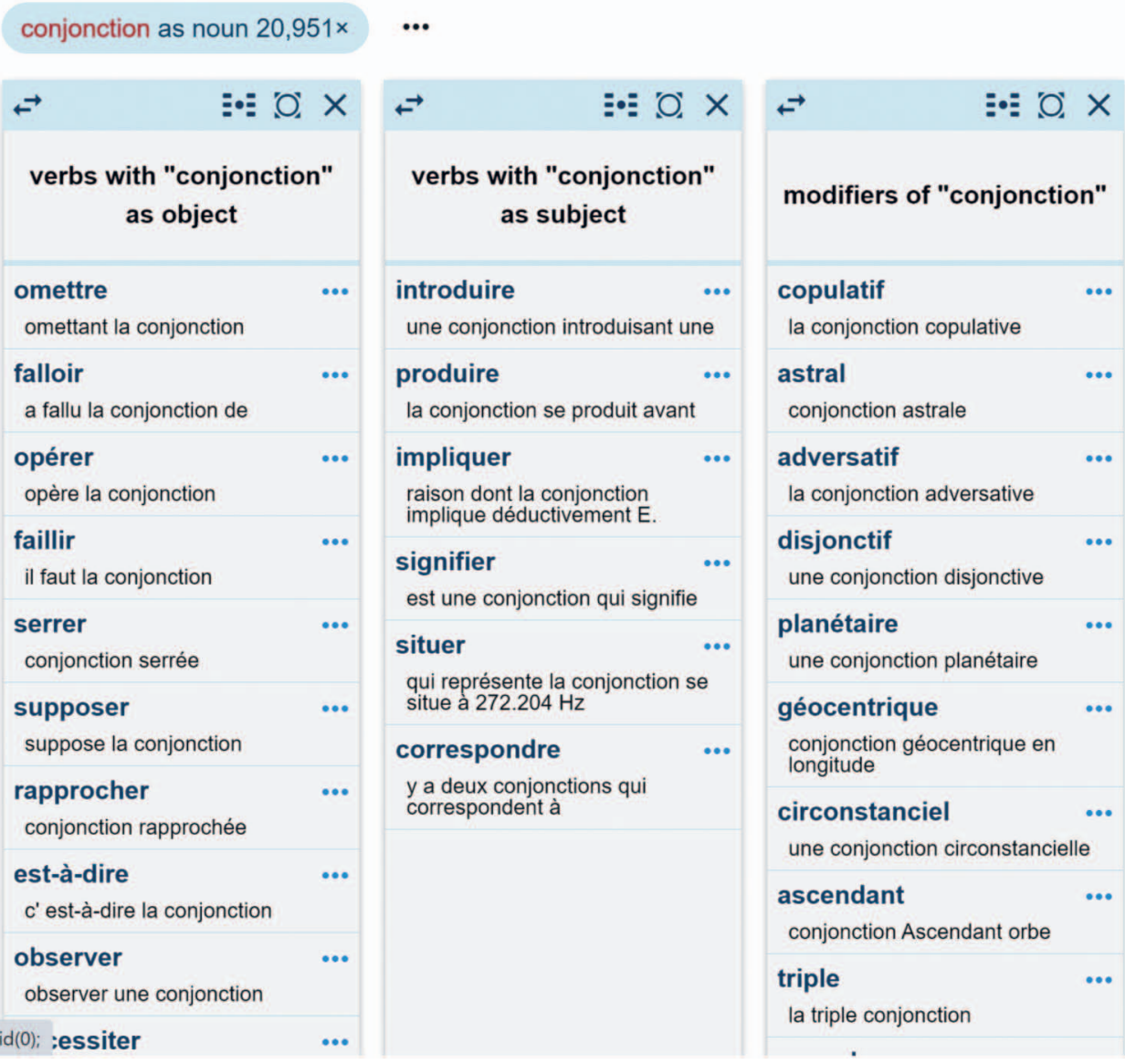




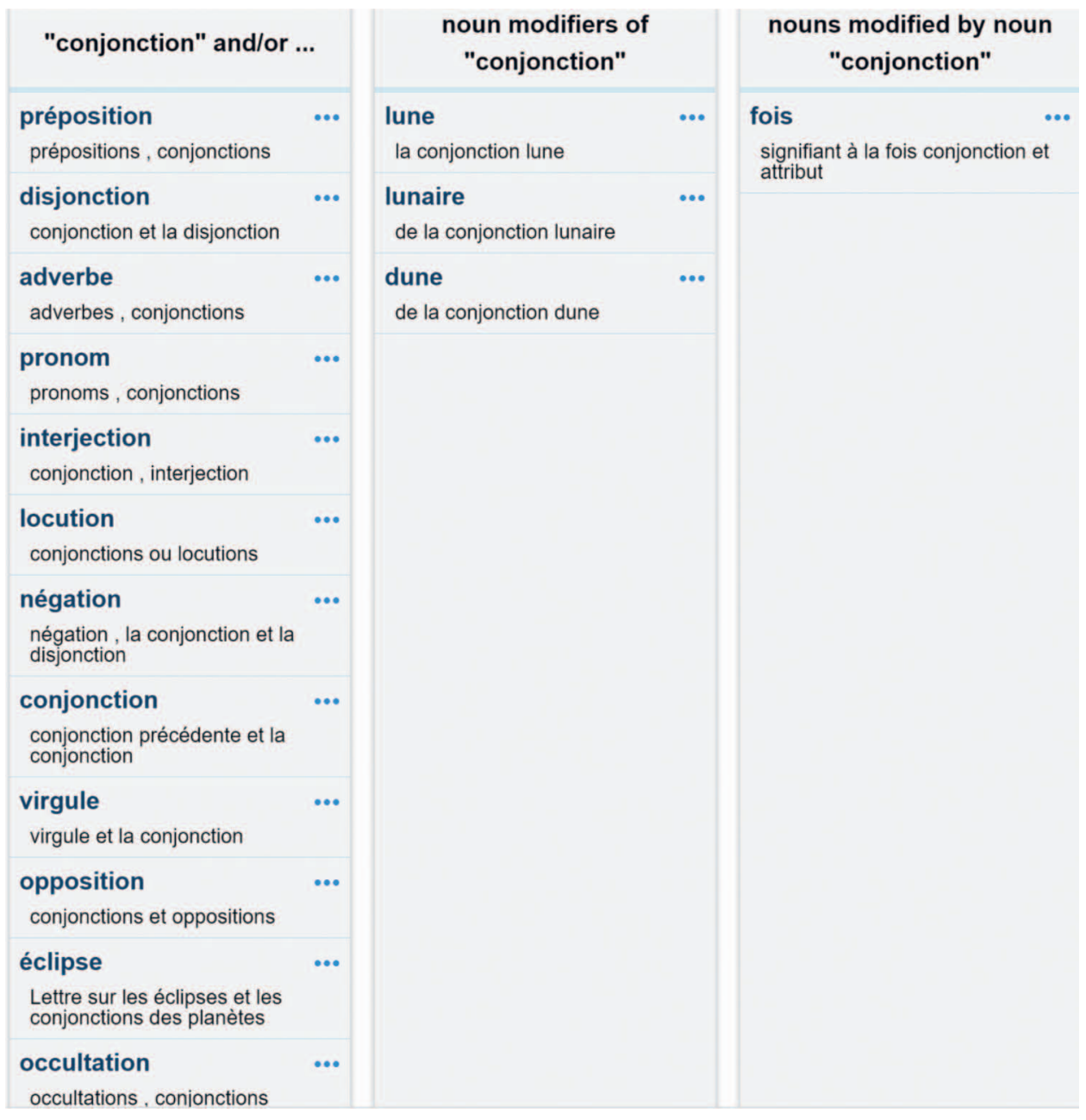

À travers les exemples des opérateurs et attributs, appropriés aux paires des mots : conjonction - spójnik et conjonction - koniunkcja et listés dans les tableaux 1, 2, 3 et les tableaux de Sketch Engine, nous avons observé qu'ils assureraient la bonne traduction du mot en question. Cependant, en ce qui concerne les opérateurs de conjonction - koniunkcja, nous n'en avons trouvé aucun qui activeraient cette traduction de façon directe. Tous les opérateurs qui s'y appliquent sont de nature générale (cf. p. ex. la notion d'opérateurs généraux et appropriés dans la conception des classes d'objets de G. Gross, 1994, 2008, 2012 ; cf. aussi W. B a n y ś, 2018 ; A. C ze kaj, 2014), c'est-à-dire qu'ils peuvent activer plus qu'un sens du mot en question et qui peuvent aller de pair avec beaucoup de classes d'objets. Ainsi, les opérateurs, tels que p. ex. : observer, reconnaître, définir, examiner, voir, comprendre, traduire, trouver, chercher, omettre, rapprocher, 
supposer, etc., qui accompagnent souvent le mot analysé dans beaucoup de textes, pourraient indiquer en même temps tous les équivalents polonais possibles. Ce phénomène ne paraît pas trop étonnant surtout que le mot conjonction, représentant un nom d'action ou un nom résultatif qui vient du verbe conjoindre, possède les sens liés soit à l'action de joindre un élément à l'autre soit à l'état d'être le résultat de conjoindre quelque chose à quelque chose d'autre. Il est donc évident que presque tous les opérateurs qui vont de pair avec cette unité lexicale seront ceux qui sont utilisés d'habitude avec les noms d'action ou les noms résultatifs de ce type. Nous n'en avons trouvé que quelques-uns qui sont caractéristiques pour conjonction - spójnik et qui pourraient activer cette traduction-là, mais ils ne sont pas nombreux (cf. tableau 2).

À part les opérateurs du type général, il y a aussi des attributs, accompagnateurs fréquents de conjonction, qui ne joueraient pas non plus le rôle des activateurs des sens précis, p. ex. rapide, possible, logique, négative, dernière, première, mauvaise, belle, magnifique, ancienne, etc. Alors, parallèlement, ce type d'attributs généraux ne conduirait pas le programme à différencier automatiquement un sens du mot de l'autre.

Et c'est en ce moment que viennent en aide les cadres et/ou les scripts, outils efficaces, grâce auxquels il serait possible de résoudre de manière plus ou moins préférentielle le problème de polysémie du mot en question. La distinction de conjonction - koniunkcja (au sens astronomique et logique) de conjonction spójnik (au sens grammatical) ne paraît pas très problématique, si l'on se sert des mots-activateurs placés dans les contextes proches ou plus lointains du mot en question. Pour décrire donc les cadres et/ou les scripts qui conditionneraient telle ou telle traduction, il suffit de lister les mots qui sont sémantiquement proches au mot analysé et qui constituent son champ lexical.

Voilà à cet égard quelques exemples de contextes qui permettent d'observer le rôle des mots-activateurs de sens dans le processus de désambiguïsation :

(1) Le plus facile à se représenter est la conjonction.

Lorsque deux planètes se superposent sur la carte astrologique, elles occupent la même position, leur écart angulaire est alors de $0^{\circ}$ (...).

Lorsque deux planètes sont très proches sur un thème astral, leurs énergies et leurs influences sont associées de manière neutre, le résultat dépendra des affinités ou des inimitiés des planètes en présence. (https://www.alexandrejohan.com/articles/comprendreSonThemeAstral, consulté le 25 avril 2020)

(2) Le schéma ci-dessus montre une planète extérieure (la planète en rouge). Selon la position de cette planète par rapport à la Terre (en bleue) et au Soleil (en jaune), on parle de conjonction, d'opposition ou de quadrature. Lorsqu'une planète est en opposition le Soleil, la Terre et la planète sont allignés dans cet ordre. Vu de la Terre, la planète se trouve dans la direction opposée au Soleil. (http://astro.vision.free.fr/ opposition_mars_2003.php, consulté le 25 avril 2020) 
(3) Nous avons vu dans l'article quand utiliser le subjonctif, que nous utilisons en français de nombreuses conjonctions pour exprimer la concession, le but, l'antériorité, la condition, etc. Certaines sont suivies du subjonctif, d'autres de l'indicatif. (...) (https:// parlez-vous-french.com/bien-que-subjonctif-ou-indicatif/, consulté le 25 avril 2020)

(4) Mais en fait, si hypothétique n'exclut pas seulement le conditionnel, donc la recette - qui est un moyen pédagogique de sténographier une règle de grammaire - est incomplète; la conjonction ne se combine en effet pas non plus avec le futur, futur morphologique, comme dans.... (https://www.unil.ch/clsl/files/live/sites/clsl/files/shared/clsl13.pdf, consulté le 25 avril 2020)

Les exemples cités ci-dessus montrent comment les mots du même champ sémantique qui se trouvent dans le voisinage de conjonction activent sa traduction. Dans les textes (1) et (2) nous avons affaire aux mots appartenant au cadre « astrologie », p. ex. : planètes, Terre, Soleil, carte astrologique, thème astral et ils influenceraient la traduction de conjonction - koniunkcja et, parallèlement, dans les textes (3) et (4), les mots-activateurs font partie du cadre " grammaire » et ils mèneraient le programme à la traduction de conjonction comme spójnik, p. ex. : indicatif, subjonctif, futur, règle de grammaire, condition, etc. Afin de décrire ces deux cadres, il suffit de faire des listes des unités sémantiquement liées à deux champs lexicaux mentionnés. À l'heure actuelle la description de ce type est faisable de manière presque automatisée grâce aux très bons outils électroniques d'analyse de corpus et aux dictionnaires qui y sont intégrés où différentes relations sémantiques entre les mots sont décrites, p.ex. synonymie, antonymie, analogie, champs lexicaux, collocations, etc. (p. ex. Antidote, CNRTL, Sensagent, WordNet pour l'anglais, Sketch Engine). Et, en cas de doute quant au choix de l'équivalent adéquat entre deux langues, le programme pourrait se servir de la base de données ainsi construite pour « décider » de la traduction appropriée.

Google Translator proposant de traduire convenablement conjonction du texte (3) et (4) comme koniunkcja et spójnik respectivement, dans ce dernier exemple, (4), le module de traduction est moins adéquat donnant la traduction fautive $k o$ niunkcja, cf. :

Ale jeśli hipotetyczny nie wyklucza tylko warunku, to przepis - który jest edukacyjnym sposobem stenografowania reguły gramatycznej — jest niekompletny; koniunkcja nie łaczy się także z przyszłościa morfologiczna... (Google Translator, consulté le 15 avril 2020)

Analysons dans ce contexte encore quatre textes suivants :

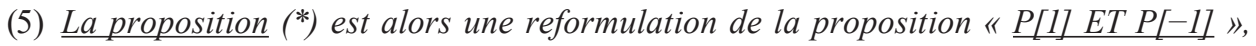
obtenue en faisant opérer le connecteur logiqueET sur les deux propositions P [1] 
et P [-1], c'est-à-dire en construisant leur conjonction. On passe de la proposition " 1 est une solution réelle de l'équation ?! - $1=0$ ET -1 est une solution réelle de l'équation ? 2 ! $1=0 »$ à la proposition (*) par des manipulations de la langue française : tout d'abord une contraction par mise en facteur de " est une solution réelle de l'équation ?! $-1=0$ », puis accord du verbe et de l'attribut qui doivent être mis au pluriel (https://hal.archives-ouvertes.fr/hal-01570177/document, consulté le 25 avril 2020)

(6) Un connecteur est un opérateur sur les propositions. Le connecteur NON opère sur une proposition $P$, et permet d'obtenir sa négation $N O N(P)$, les connecteurs ET, OU, IMPLIQUE, ÉQUIVAUT À opèrent sur deux propositions $P, Q$, et permettent d'obtenir la conjonction $(P$ ET $Q)$, la disjonction $(P$ OU $Q)$, l'implication $(P \Rightarrow Q)$ et l'équivalence $(P \Leftrightarrow Q)$. Cet aspect syntaxique des connecteurs est important dans la distinction entre et propositionnel et et couple par exemple (https://hal.archives-ouvertes.fr/hal-01570177/document, consulté le 25 avril 2020)

(7) On s'aperçoit très vite que Bastide décrit la valeur du cadrage dans la production d'un objet cognitif à travers des procédures qui nous rappellent les opérations rhétoriques fondamentales, ces dernières étant communes aux deux rhétoriques mentionnées plus haut (l'addition, la suppression, la substitution, la dissociation, la conjonction, etc.) (https://www.erudit.org/en/journals/pr/2010-v38-n1-pr3858/039701ar/, consulté le 25 avril 2020)

(8) Quintilien propose de comprendre la figure comme un changement raisonné du sens ou du langage par rapport à la manière ordinaire et simple de s'exprimer. Il fait à nouveau un parallèle avec le corps humain: prise au sens restreint, la figure lui apparaît semblable à l'attitude volontaire que peut prendre un corps (il est debout, assis ou couché...). Dans cette acception la figure apparaît donc comme un écart délibéré par rapport à une norme de discours. Cette définition a été indéfiniment reprise depuis Quintilien. Elle n'en pose pas moins de nombreux problèmes qui menacent sa cohérence. Elle conjoint un critère psychologique (la figure est une opération volontaire), un critère socio-linguistique (la figure s'écarte d'une norme de discours) et un critère formel (la figure s'écarte de la forme la plus simple du discours). Or chacun de ces critères prête à discussion. Et leur conjonction est problématique (https://www.unige.ch/lettres/framo/enseignements/methodes/frhetorique/fr012000. html, consulté le 25 avril 2020)

Dans les textes ci-dessus conjonction devrait être traduite comme koniunkcja. Les cadres « logique » et « rhétorique » indiqueront cette traduction à l'aide des mots-activateurs qui sont employés dans son voisinage. Ainsi, si l'on parle de conjonction - koniunkcja au sens logique du mot, on observe dans son entourage plus ou moins proche les mots qui y sont caractéristiques, tels que p. ex. disjonction, équivalence, implication, logique, connecteur " et », équation, etc. 
(cf. textes : (5), (6)). Il est aussi important de souligner que dans ce type des textes apparaissent très souvent des formules logiques représentant certains phénomènes et elles faciliteraient aussi l'interprétation de sens porté par le mot analysé, p. ex. : $P, Q, P$ et $Q$, etc. (cf. textes : (5), (6)). Par contre, les fragments (7) et (8) comportent les mots typiques pour le cadre " rhétorique », p. ex. figure, norme de discours, forme du discours, cohérence, substitution, suppression, addition, dissociation, opérations rhétoriques, etc., ce qui constitue un bon indice pour choisir un équivalent polonais préféré.

Il arrive parfois aussi que conjonction - spójnik est employée dans le contexte " rhétorique ", mais dans ce cas-là elle est d'habitude accompagnée d'attributs de type : de coordination, de subordination, etc. (cf. texte (9)) ou de conjonctions grammaticales énumérées dans la même phrase (cf. texte (10)). Ce sont donc de tels indices qui aident suffisamment à désambiguïser le sens (cf. le tableau 2). Par exemple :

(9) L'ellipse est utile dans la formation de termes : voiture (automobile). La suppression de la conjonction de coordination donne l'asyndète, la suppression de la conjonction de subordination forme la parataxe, et de celle du verbe résulte la phrase nominale. Dans le langage poétique elle ajoute à la vigueur d'expression et donne une force soutenue à l'élan de la parole; elle évite les lourdeurs. En mettant des structures essentielles à nu, elle ajoute aussi à la clarté : (Il y a des) "Murmures» (Michel Butor). Elle peut marquer des émotions fortes; ainsi dans l'Andromaque de Racine éclate toute l'amertume d'Hermione envers Pyrrhus : "Je t'aimais inconstant : qu'auraisje fait fidèle? "C'est le contexte qui éclaire cette ellipse " hardie " (Laharpe). Grâce à celui-ci, le lecteur comprend : "Je t'aimais (quoique tu fusses) inconstant, qu'aurais-je fait (si tu étais) fidèle? " Plus on est rebuté par l'expression complète et plus on apprécie l'économie de l'ellipse (https://www.universalis.fr/encyclopedie/ ellipse-rhetorique/, consulté le 25 juin 2020)

(10) Je n'ai pas l'intention, ici, de discuter cette position - quelque forme qu'elle prenne. Je voudrais seulement illustrer l'utilisation qui y est faite des lois de discours, instrument puissant (et auquel on peut même reprocher, comme aux transformations de la grammaire generative, de l'être trop). Un premier exemple, emprunté à Cornulier 1978. Il m'est arrivé (Ducrot 1972, chap. 6) de donner pour fonction fondamentale à la conjonction si de permettre un « acte de supposition ». On demande au destinataire d'imaginer telle ou telle situation, et, une fois qu'on l'a placé dans cette situation fictive, on fait une assertion, une interrogation, un ordre... etc. Un avantage de cette proposition est qu'elle rend compte de ce qu'on a appelé le "si d'énonciation 》 par opposition au " si implicatif » (http://www.utc.fr/ jguignar/SC21/Textes/ Ducrot\%20(Maximes,\%20Discours).pdf, consulté le 15 juin 2020)

Et dans le cas du texte ci-dessous (11) la situation est aussi intéressante, puisque, bien que conjonction apparaisse dans un contexte général " rhétorique », l'emploi spécifique du mot dans le contexte précis, comme partie principale de la 
locution prépositive à la conjonction de, nous la fait traduire comme połaczenie et non pas koniunkcja.

(11) Cela étant, une telle démonstration repose sur deux présupposés qui n'ont rien d'évident : d'une part, l'association de l'examen détaillé à un projet d'interprétation, d'autre part l'assimilation de l'analyse des régularités fonctionnelles à un travail dans la transtextualité.

À la conjonction de ces deux présupposés intervient implicitement le statut idéologique du texte : dans la mesure où son identité est interprétée en termes d'unicité et d'infinitude métaphysiques (ou esthétiques), il interdit par préjugé une analyse qui l'aborderait en termes de spécificité rationnelle, c'est-à-dire d'agencement modélisable de traits, procédures ou fonctionnements typiques (https://www.fabula.org/ lht/3/noille.html, consulté le 25 avril 2020)

En observant le comportement linguistique de conjonction, nous sommes tombés encore sur trois exemples intéressants :

(12) Le critère des "faits établis au-delà de tout doute raisonnable " utilisé de longue date par la Cour en matière de preuve ne doit pas être remplacé par un aperçu impressionniste des éléments de preuve. De même, le critère substantif du « contrôle effectif » ne doit pas être dilué pour les besoins de l'affaire. Le présent arrêt restera donc comme un exemple malheureux de conjonction négative d'inertie judiciaire, de manque de preuves, de faits omis et de dilution des critères juridiques établis (...) (https://hudoc.echr.coe.int/fre\#\{\%22itemid\%22:[\%22001-155656\%22]\}, consulté le 25 avril 2020)

(13) Le fait d'analyser pour pas que comme une locution conjonctive n'explique malheureusement pas pourquoi pour pas que est la seule conjonction négative de la liste, ni d'ailleurs pourquoi il n'y a pas un déplacement pareil de pas, là où une subordonnée négative est facilement envisageable (*malgré pas que, *vu pas que, *à cause pas que) (https://www.cambridge.org/core/journals/journal-of-french-language-studies/ article/pour-ne-pas-que-une-locution-conjonctive-entre-normes-et usages/121A4EF6 7369A43FBDE1294089C1B001/core-reader, consulté le 25 avril 2020)

(14) Toutefois, à partir d'octobre 2014 et jusqu'en mai 2015, François Hollande bénéficiera d'un peu de chance (Jupiter en conjonction à son Soleil), même si le climat général restera très négatif pour lui : sa Lune natale sera transitée par une conjonction négative de Pluton, un carré par $\underline{\text { Uranus }}$ et un demi-carré par Saturne au premier semestre 2015... (http://astrologie277.rssing.com/chan-30200089/all_p5.html, consulté le 25 avril 2020)

$\mathrm{Vu}$ que l'adjectif « négative » qui accompagne le mot conjonction dans tous les exemples ci-dessus n'est pas distinctif, il est nécessaire de chercher une information distinctive dans les phrases qui le précèdent et/ou qui le suivent. Ainsi, 
dans le texte (13) nous pouvons observer des mots-activateurs du cadre " grammaire », donc la traduction de l'expression serait dans ce cas-ci spójnik negacji, et, par contre, dans le texte (14) les mots-activateurs appartiennent au cadre « astrologie ", d'où la traduction polonaise koniunkcja negatywna (tous les mots-activateurs sont soulignés dans les textes). Cependant, dans l'exemple (12) nous avons proposé l'équivalent polonais negatywne połaczenie. Le contexte dans lequel conjonction négative a été employé n’est pas ni de type « grammaire », ni «astrologie », ni « logique », ni « rhétorique » non plus. Nous avons d'ailleurs trouvé beaucoup d'exemples où conjonction apparaît dans des contextes différents de ceux que nous avons dégagés plus haut. Alors, l'une des questions générales que l'on doit se poser concerne la traduction des mots dans des contextes qui ne sont pas distinctifs. Une autre question à se poser est de savoir si nous avons proposé le bon équivalent polonais dans le contexte en question - negatywne połaczenie. Peut-être y a-t-il encore une autre possibilité de traduire cette expression, p. ex. negatywna koniunkcja?

À cet égard il est intéressant de voir les traductions fautives des fragments (13) et (14) ci-dessus, proposées par Google Translator (consulté le 15 juin 2020) :

Traduction de (13):

Fakt, że nie jest to spójna lokalizacja, nie wyjaśnia niestety, dlaczego nie jest to jedyna ujemna koniunkcja listy, a ponadto, dlaczego nie występuje podobne przesunięcie kroków gdzie negatywny podwładny jest łatwo możliwy (* pomimo tego, * tego nie widziałem, * nie dlatego, że).

Traduction de (14):

Jednak od października 2014 r. do maja 2015 r. François Hollande skorzysta z odrobiny szczęścia (Jowisz w połaczeniu ze swoim Stońcem), nawet jeśli ogólny klimat pozostanie dla niego bardzo negatywny: jego rodzimy Księżyc będzie tranzytem przez ujemna koniunkcje Plutona, kwadrat Urana i pót kwadratu Saturna w pierwszej polowie $2015 \mathrm{r}$.

Après avoir analysé beaucoup de textes avec le mot conjonction et d'autres qui présentent le même type de " confusion », il paraît que dans ce cas-là il y a deux propositions de traduction à choisir :

a) plutôt koniunkcja que połaczenie,

b) plutôt połaczenie que koniunkcja.

Il faut encore ajouter que le mot conjonction dans ces contextes est suivi d'habitude soit de prépositions « de », soit de préposition « entre », de type, p. ex. : la conjonction de....... (et de......., etc.) ou la conjonction entre ......... (et ..........., etc.).

Pour mieux voir encore la complexité des choix, regardons les exemples cidessous : 
(15) Mais de nombreuses limites ont surgi. P Leroux plus tard effectuera la conjonction de l'impulsion utopique et de la question politique, en l'occurrence de la démocratie, en donnant forme à l'attraction grâce à un principe fondamentalement politique, à savoir l'amitié, politique de la philia contre les politiques d'éros aussi bien prônées par Fourier que par les saint simonien. L'amitié a la particularité d'instaurer un lien dans la séparation, un lien qui se noue tout en préservant un espace entre les membres de la communauté et met à distance donc toute tentation de communauté fusionnelle (https://ddloeil.github.io/rencontre\%202/jekyll\%20update/2018/02/21/ utopie/, consulté le 25 avril 2020)

(16) À partir d'une étude de la fonction imaginale chez l'homme comme étant la conjonction de deux principes, l'un passif, la mémoire, et l'autre actif, l'imagination, cet article montre comment, dans le domaine des images fabriquées, ce qui définit l'imaginaire collectif vient prendre progressivement la place de l'imaginaire individuel. L'effet paradoxal des images est de priver l'individu des siennes, la salutation des médias visuels d'images n'engageant pas l'imagerie individuelle, mais plutôt une action (https://www.erudit.org/en/journals/cine/1994-v4-n3-cine1502289/1001037ar/, consulté le 25 avril 2020)

(17) Cette situation résulte principalement de la conjonction de deux facteurs. D'abord, même si le transport est juridiquement et techniquement faisable, il reste difficile en raison des conditions de transport actuelles telles qu'elles sont décrites aux considérants 22 à 29. Ensuite et surtout, les clients sont liés à leur fournisseur actuel par des contrats de longue durée (https://eurlex.europa.eu/LexUriServ/LexUriServ.do?u ri=OJ:L:2003:248:0051:0061:FR:PDF, consulté le 25 avril 2020)

(18) Le projet

Chais'Art vient de la conjonction de l'Art et de la chaise roulante et de la volonté de décliner le sigle international de l'accessibilité aux personnes handicapées à travers différents thèmes et illustrations suscitant tantôt la dérision, l'étonnement, l'interrogation.

Ses objectifs sont multiples:

Communiquer sur le thème du handicap, afin de...

- Démystifier celui-ci aux yeux du grand public

- Sensibiliser la population à la situation des personnes handicapées sur divers aspects de la vie courante

- Créer du lien entre les personnes, quelle que soient leurs situations

- Dénoncer le manque d'inclusion et d'accessibilité dans le domaine public, et militer contre ces injustices.

- Créer un support graphique aussi complet que varié, avec un humour et accessible au plus grand nombre (https://sites.google.com/site/chaisart1/projet, consulté le 25 avril 2020) 
(19) Cette histoire d'Arkema qui s'est remis à l'endroit tout seul, c'est complètement incroyable mais on n'a pas le choix, il faut y croire. Quelqu'un qui serait venu sur zone en douce, qui l'aurait retourné, j'y crois moins. C'est le hasard, le miracle, donc: la conjonction d'une vague énorme, d'un coup de vent, d'autres choses. Et le flotteur qui s'était rempli d'eau, pour aider au retournement. Avec des moyens techniques, ça aurait été une galère. Magique! (https://voilesetvoiliers.ouest-france. fr/course-au-large/route-du-rhum/route-du-rhum-mon-rhum-a-moi-par-jean-le-cam229a47e0-f7ed-11e8-88f8-2d6a6186alcc, consulté le 25 avril 2020)

(20) Spécialités :

- Riz Chikara de Tateyama au goût unique façonné par la conjonction de l'eau pure, de la terre riche et des vents de montagne qui font varier la température; entièrement cultivé à la main.

- Eau naturelle de Tateyama

- Bouillie de riz de Tateyama (http://toyama-muslim.jp/fr/restaurant/yoshimineyurando-tateyama-corp-boutique-du-centre-cultutel-yoshimine/, consulté le 25 2020)

Puisque, dans les exemples cités ci-dessus, les cadres, même s'ils sont différents, ils ne sont pas distinctifs, il faudrait donc se référer à d'autres indices qui faciliteraient le choix de l'équivalent convenable. Dans les cas comme ceux-ci, on doit avoir recours à l'analyse du registre de la parole/du texte qui pourrait apporter une information nécessaire (cf. à cet égard un travail de D. B ib e r et S. Con ra d, 2019 et de M. Sas s i e r, 2008). Ainsi, on remarque que les textes (15) et (16) sont écrits dans le registre scientifique, ce qui est visible grâce à l'emploi d'un grand nombre de mots savants, donc l'équivalent de conjonction serait préférentiellement koniunkcja que połaczenie (cf. a). Par contre, les textes (19) et (20) abordent les sujets « quotidiens », le registre est standard et il serait étrange, mais certainement non pas exclu, de choisir cette première option de traduction koniunkcja. Il serait mieux de proposer ici préférentiellement l'équivalent polonais połaczenie à la place de koniunkcja (cf. b), et, c'est cette traduction-là, qui, d'après nous, serait choisie dans ce type des textes. En ce qui concerne les fragments (17) et (18), ils sont écrits dans le registre standard, comme les textes précédents, mais leurs styles n'étant ni scientifique, ni familier et leurs sujets étant loin d'être « quotidiens », les deux possibilités de traduction y seraient acceptables. Vu que le programme aurait sans aucun doute un grand problème de distinguer les deux derniers types de textes, il serait raisonnable d'introduire un critère préférentiel supplémentaire à suivre : si le texte est de caractère scientifique - la traduction serait koniunkcja et dans d'autres types de textes ce serait połaczenie. Il est quand même à remarquer que, dans ce type des situations, le locuteur peut avoir recours, pour une raison ou une autre, à différents jeux linguistiques, voulant paraître 
p. ex. plus savant qu'il l'est en réalité, en introduisant un mot du registre plutôt savant dans une conversation quotidienne et ne touchant pas les questions scientifiques, et c'est pourquoi le recours à une traduction préférentielle, permettant de rendre compte de cette créativité et flexibilité de langue, qui nous est naturelle, est absolument indispensable.

Remarquons aussi que la situation peut être encore plus complexe, cf. p. ex. :

(21) Avec la reproduction, nous sommes devant un fait de nature : un nouvel être humain ne peut résulter que de la conjonction d'un ovocyte et d'un spermatozoïde, produits physiologiques de deux individus de sexes différents. Mais si l'état des connaissances ne permit pas pendant longtemps de distinguer l'individu des constituants de la reproduction, aujourd'hui il est possible d'isoler ces éléments, donc d'engendrer en dehors de la rencontre effective d'un homme et d'une femme. Il peut y avoir soit fécondation in vitro ou médicalement assistée (PMA), soit insémination artificielle avec donneur (IAD), soit mère porteuse qui prête son utérus (GPA) (https://www. cairn.info/revue-nouvelle-revue-de-psychosociologie-2013-1-page-279.htm, consulté le 25 avril 2020)

(22) Il va de soi que nous avons à privilégier la lecture pour laquelle a opté Averroès. A la lecture qui agit sur la ponctuation, en différant le point afin que la phrase continue pour assurer la conjonction entre Dieu et les savants parmi les humains capables d'interpréter la part obscure de la Révélation. Ce privilège de l'interprétation mérite d'être rappelé, c'est un enjeu pour les trois monothéismes, (il concerne surtout la Bible et le Coran), afin de conjurer la dimension guerrière, la part belliqueuse, ce que les musulmans appelleront par la suite "Djihâd » parce que cette association entre le Djihâd et la guerre légale n'existe pas explicitement dans la lettre coranique, elle est en puissance, sa construction est possible.

Malgré le fait que les textes (21) et (22) sont écrits dans le registre scientifique ou quasi scientifique, conjonction y serait traduite décidément plutôt, mais toujours plutôt, comme połaczenie et non pas koniunkcja. Cette plus grande force préférentielle, sans être impérative, est due au fait que les concordances de la conjonction d'un ovocyte et d'un spermatozoïde et la conjonction entre Dieu et les savants constituent en fait presque les modifieurs, les attributs, conventionnels quasi appropriés de conjonction. Mais cette façon de parler pourrait être changée dans un jeu linguistique du locuteur et conjonction pourrait être traduite comme koniunkcja si l'interprétateur le décide. De ce point de vue, quand nous parlons du caractère préférentiel des traductions, nous appliquons dans nos analyses davantage une logique floue, discontinue, basant davantage sur les valeurs $+/$ - , plus ou moins, probabilistes, que sur celle du vrai ou faux absolus et sur les décisions absolues. Conscients de toute la complexité de l'interprétation et de la traduction des textes, nous avons recours aux outils et analyses mentionnés. D’autre part, puisque les programmes de traductions nécessitent une instruction claire com- 
ment procéder, nous indiquons, guidés par les principes ci-dessus, les traductions préférentielles des mots et des textes et nous pouvons ainsi instruire le programme comment appliquer les principes de traduction.

En naviguant sur différents corpus, nous avons découvert encore une autre possibilité de traduire le mot analysé. Il s'avère que conjonction dans le cadre « mariage » signifie en polonais związek, mais cet emploi apparaît, dans presque tous les cas, dans les textes vieillis. Les attributs qui l'accompagnent et qui indiquent de manière directe cette traduction sont les suivants, p. ex. :

Attributs :

$$
\text { conjonction }(f) \text { - związek }
$$

\begin{tabular}{|l|l|}
\hline conjonction adultère & zwiazek cudzołożny \\
\hline conjonction alternative & związek alternatywny \\
\hline conjonction charnelle & związek cielesny \\
\hline conjonction conjugale & związek małżeński \\
\hline conjonction de deux personnes & związek dwóch osób \\
\hline conjonction de mariage & związek małżeński \\
\hline conjonction d'un homme et d'une femme & związek mężczyzny i kobiety \\
\hline conjonction hétérosexuelle & związek heteroseksualny \\
\hline conjonction homosexuelle & związek homoseksualny \\
\hline conjonction hors du mariage & związek pozamałżeński \\
\hline conjonction illégitime & związek niezalegalizowany \\
\hline conjonction illicite & związek nielegalny \\
\hline conjonction maritale & związek małżeński \\
\hline conjonction légitime & zwiazek zalegalizowany \\
\hline conjonction matrimoniale & związek małżeński \\
\hline conjonction mystique & związek mistyczny \\
\hline conjonction physique & związek fizyczny \\
\hline conjonction romantique & związek romantyczny \\
\hline conjonction sacramentale & związek sakramentalny \\
\hline conjonction sexuelle & związek seksualny \\
\hline etc. & \\
\hline
\end{tabular}

\section{Par exemple :}

(23) Hormis ses deux derniers paragraphes, l'article est donc un copié-collé, à de minuscules variantes près, du Dictionnaire de la Bible (1722-1728) de Dom Calmet, qui définit sans surprise l'inceste comme la " conjonction illicite entre des personnes qui sont parentes jusqu'aux degrés prohibés par les lois de Dieu ou de l'Église ». Si la définition se fonde sur la lecture des textes sacrés (Lévitique, 18, 1-18) ou des écrits de Pères de l'Église, ce qui apparaissait dans l'ouvrage de Calmet, la reprise 
dans l'Encyclopédie gomme la référence à ces sources (https://journals.openedition. org/rde/5392?lang=en, consulté le 25 avril 2020)

À la fin de l'analyse, il est intéressant de s'arrêter encore sur la traduction de l'expression française, figée, très souvent employée, en conjonction avec... . On pourrait traduire cette expression en polonais de deux manières : $w$ połaczeniu $z$.... et $w$ koniunkcji z...... et cette traduction dépend naturellement du contexte. Par exemple :

(24) Lot de 2 boules en verre avec ouverture et suspension. Pour peindre avec la couleur de la fenêtre ainsi qu'à des fins décoratives avec des fleurs, de petits bonbons et d'autres choses mignonnes. Peut également être utilisé comme lanterne en conjonction avec des bougies chauffe-plat. Avec la couleur effet gel les boules créent une atmosphère de noël merveilleuse.

En verre transparent, avec une ouverture demi-face

Couleur: Transparent

Matière: verre

Contenu du paquet: 2 x bougeoirs (https://fr.aliexpress.com/item/4000277523951.

html, consulté le 25 avril 2020)

(25) Le réchauffement global, en conjonction avec la négligence industrielle, a provoqué l'une des pires catastrophes écologiques de la fragile région arctique. Environ 23000 tonnes de diesel échappées du réservoir d'une centrale électrique ont contaminé la péninsule de Taïmyr, d'une superficie égale à dix fois celle de la Suisse (https://www.letemps.ch/sciences/maree-noire-norilsk-une-catastrophe-annoncedautres, consulté le 25 avril 2020)

(26) Une planète est en conjonction avec le Soleil si elle est dans la même direction que lui. Elle est bien sûr inobservable autour des dates de conjonction sauf cas particulier, celui où la planète passa devant le Soleil, entre lui et nous par conséquent. Cela ne peut donc arriver qu'aux planètes inférieures, lors des conjonctions dites " inférieures " (voir schéma) (https://www.clicanoo.re/Zot-Clicanoo/Article/2007/02/11/ Astronomie-Elongations-oppositions-et-conjonctions_71009, consulté le 25 avril 2020)

(27) Substitution de $x 2$ par a $C^{\prime} 3=\neg F P E(a) V \neg E M(a)$ Substitution de $x 1$ par a $C^{\prime} 2=$

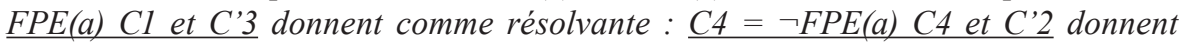
comme résolvante une clause vide... Il est donc impossible de satisfaire la négation de la conclusion en conjonction avec les prémisses, donc la conclusion est une déduction logique correcte (https://perso.liris.cnrs.fr/marie.lefevre/ens/BIA/BIA2019TD3-Logique-correction.pdf, consulté le 25 avril 2020)

(28) Au plan discursif, Benveniste oppose le plan de l'histoire. Le je ramène inévitablement à l' "ici-maintenant" de l'énonciation, et ce même lorsqu'il exprime une temporalité révolue (j'allais, je faisais, etc.) ; par contre, la troisième personne, en 
conjonction avec certaines formes de l'expression temporelle du passé, engage pour Benveniste le plan historique, et par extension, le plan privilégié de la fiction écrite. Il définit le récit historique comme "le mode d'énonciation qui exclut toute forme linguistique 'autobiographique'. L'historien ne dira jamais je ni tu, ni ici, ni maintenant, parce qu'il n'empruntera jamais l'appareil formel du discours ». À ce stade, il nous apparaît fécond de caractériser la configuration énonciative du jeu par un système mixte (http://www.webbynerd.com/artifice/dossierarchives/148.html, consulté le 25 avril 2020)

Puisqu'il y a généralement trois situations où conjonction soit traduite comme koniunkcja dans cette expression, il suffirait de déterminer trois cadres correspondants pour les indiquer précisément. Il s'agit des textes (26), (27), (28) où les mots-activateurs (soulignés dans les textes) venant des cadres « astrologie », " logique » et « rhétorique » activeraient la traduction koniunkcja. Dans les autres cas on pourrait préférentiellement proposer l'équivalent polonais połaczenie (textes (24), (25)), parce que les cadres n'arriveraient pas à différencier ces deux sens.

L'analyse effectuée selon la Méthode Orientée Objets fait bien voir la complexité de traduction des unités linguistiques polysémiques. La description de ce type est d'autant plus importante qu'elle devrait servir de base de données lexicales sur laquelle opérerait un programme de traduction ayant pour but de traduire automatiquement des textes d'une langue vers l'autre. En cherchant une équivalence sémantique du français vers le polonais à travers l'exemple de conjonction, nous avons observé que parmi différents sens du même mot polysémique il y en a ceux qui ne sont pas trop problématiques à décrire, mais il y en a aussi d'autres qui exigent beaucoup plus de minutie descriptive, parce qu'il est plus difficile d'indiquer précisément leurs correspondances sémantiques. Les cadres et/ou les scripts aident souvent avec efficacité à accomplir cette tâche, mais il y des cas où, pour bien traduire le mot, il faut se référer encore p. ex. au registre ou au domaine, et, c'est ainsi que l'on trouve des indices indispensables pour la traduction. Et parfois, même si l'on essaye de préciser les emplois des mots de manière la plus complète possible, on n'est pas capable de prévoir toutes les situations langagières où un mot donné pourrait apparaître, et, ce qui en résulte, de prévoir toutes ses traductions. On propose donc des règles les plus précises possible grâce auxquelles, d'une part, on pourrait éliminer de graves erreurs de traduction automatique, et, d'autre part, on pourrait indiquer au programme de traduction les pistes préférentielles basées sur les principes davantage de la logique floue, +/-, non-discrète, probabiliste, que de la logique vériconditionnelle, ce qui ne veut pas dire que, dans les cas des opérateurs et attributs appropriés très clairs et distinctifs en particulier, elles ne peuvent pas se rencontrer. C'est donc le critère de préférence ainsi entendu qui doit être pris en compte dans toutes ces situations floues et vagues. Il est aussi important de dire que le recours aux règles de traduction établies selon l'AOO basées sur la préférentialité liée à la probabilité, combinées 
avec les méthodes statistiques classiques et/ou les réseaux de neurones artificiels, dont se servent souvent les traducteurs automatiques d'aujourd'hui, permettra d'optimiser les résultats de traduction automatique des textes.

\section{Références citées}

B a n y ś W., 2002a : « Bases de données lexicales électroniques — une approche orientée objets. P a rtie I : Questions de modularité ». Neophilologica 15, 7-28.

B a n y ś W., 2002b : « Bases de données lexicales électroniques — une approche orientée objets. P a rtie II : Questions de description ». Neophilologica 15, 206-248.

B a nyś W., 2005 : «Désambiguïsation des sens des mots et représentation lexicale du monde ». Neophilologica 17, 57-76.

B a nyś W., 2018 : « Nouveaux anciens paradigmes : approche orientée objets, classes d'objets, psychologie écologique et linguistique ». Neophilologica 30, 25-41.

B iber D., C on rad S., 2019 : Register, Genre and Style. Cambridge University Press.

Czekaj A., 2011 : «Question de métonymie dans la traduction automatique ». Neophilologica 23, 136-149.

Czekaj A., 2014 : «Comment comprendre la classe d'objets ? ». Neophilologica 26, $232-244$.

C z e k aj A., 2018 : « Perception et métonymie — problèmes de traduction automatique ». Neophilologica 30, 76-88.

G offm a n E., 2010 : Analiza ramowa. Kraków, Nomos.

Gros s G., 1994 : « Classes d'objets et description des verbes ». Langages 115, 15-30.

Gros s G., 2008 : « Les classes d'objets ». Lalie 28, 113-165.

Gross G., 2012 : Manuel d'analyse linguistique. Villeneuve-d'Ascq, Presses universitaire du Septentrion.

H r a b i a M., 2016 : Itérativité dans la traduction automatique. Katowice, Wydawnictwo Uniwersytetu Śląskiego.

Fil1more Ch., 1982 : "The Frames semantics". In: The Linguistic Society of Korea (eds.): Linguistics in the Morning Calm. Seoul, Hanshin, 111-137.

Kilgariff A., 1997 : "I Don't Believe in Word Senses". Computers and the Humanities $31,91-113$.

Köve c s e s Z., 2011 : Język, umyst, kultura. Kraków, Universitas.

Min sk y M., 1985: The Society of Mind. New York, Simon and Schuster.

S a s sier M., 2008 : "Genre, registre, formation discursive et corpus ». Langage et société $124,39-57$.

Schank R., Abels on R., 1977 : Scripts, Plans, Goals and Understanding. Hillsdale, N.J., Lawrence Erlbaum.

Śmigielska B., 2013 : «Description des cadres dans l'approche orientée objets en vue de la traduction assistée par ordinateur ». Roczniki Humanistyczne LXI, Nr 8: Lingwistyka Korpusowa i Translatoryka, 49-60. 
Śmigielska B., 2015 : « Traduction automatique des mots sémantiquement proches dans l'approche orientée objets ». Neophilologica 27, 241-252.

Śm ig i e1sk a B., 2016 : «Inclusion et traduction automatique ». Language 42 : Translation and Meaning. New Serie, Vol. 2. Frankfurt, Peter Lang, 139-152.

Śmigielska B., 2017 : « De la désambiguïsation et de la traduction de "éducation" ou de l'éducation de la désambiguïsation et de la traduction ». Roczniki Humanistyczne LXV, $\mathrm{Nr}$ 8: Lingwistyka Korpusowa i Translatoryka, 157-169. 